\title{
Light Scalar and Lepton Anomalous Magnetic Moments
}

\author{
Sudip Jana, ${ }^{1}$ Vishnu P.K., ${ }^{2}$ and Shaikh Saad ${ }^{2}$ \\ ${ }^{1}$ Max-Planck-Institut für Kernphysik, Saupfercheckweg 1, 69117 Heidelberg, Germany \\ ${ }^{2}$ Department of Physics, Oklahoma State University, Stillwater, OK, 74078, USA
}

\begin{abstract}
Recent precise determination of the electron anomalous magnetic moment (AMM) adds to the longstanding tension of the muon AMM and together strongly point towards physics beyond the Standard Model (BSM). Here we present a solution to both anomalies via a light scalar that emerges from a second Higgs doublet and resides in the $\mathcal{O}(10)-\mathrm{MeV}$ to $\mathcal{O}(1)-\mathrm{GeV}$ mass range. A scalar of this type is subject to a number of various experimental constraints, however, as we show, it can remain sufficiently light by evading all experimental bounds and has the great potential to be discovered in the near-future low-energy experiments. In addition to the light scalar, our theory predicts the existence of a nearly degenerate charged scalar and a pseudoscalar, which have masses of the order of the electroweak scale. This scenario can be tested at the LHC by looking at the novel process $p p \rightarrow H^{ \pm} H^{ \pm} j j \rightarrow l^{ \pm} l^{ \pm} j j+E_{T}$ via same-sign pair production of charged Higgs bosons.
\end{abstract}

Keywords: beyond standard model, muon $g-2$, electron $g-2$

DOI: $10.31526 /$ ACP.BSM-2021.23

\section{INTRODUCTION}

Ever since the first computation of anomalous magnetic moment of the electron by Schwinger [1], it was realized that the measurement of the magnetic moments of the charged leptons can provide an accurate test of the standard model (SM) of particle physics. In the muon sector, there has been a long standing tension in the measured and predicted values of its anomalous magnetic moment $\left(a_{\mu}\right)$, which corresponds to a deviation [2]:

$$
\Delta a_{\mu}=(2.79 \pm 0.76) \times 10^{-9}
$$

which implies that the deviation has a positive sign and presents a 3.7 $\sigma$ discrepancy. On the other hand, the recently measured finestructure constant $\alpha$ using Caesium atoms [3] with unprecedented precision point towards a deviation in the electron anomalous magnetic moment $\left(a_{e}\right)$ from the experimental value as well:

$$
\Delta a_{e}=-(8.7 \pm 3.6) \times 10^{-13}
$$

This deviation corresponds to a negative $2.4 \sigma$ discrepancy. It is interesting that the deviations in the electron anomalous magnetic moment is having a opposite sign compare to $a_{\mu}$. Moreover, if the corrections to $a_{e}$ and $a_{\mu}$ are similar, then one would expect the $\Delta a_{e} \simeq 7 \times 10^{-14}$, due to the dependence on the square of lepton mass. However, from Eq. 2 its clear that this is not the case. Due to these reasons, a simultaneous explanation of these two anomalies is challenging.

In the literature, a few different mechanisms are proposed to take into account these discrepancies, e.g., by introducing new scalar states $[4,5,6]$, in the context dark matter models [7, 8] and utilizing vector-like fermions [9]. However, most of these models require additional fermion states or need to extend the gauge symmetry. Recently, we propose [10] a simple ultraviolet (UV) complete model that could address these two anomalies simultaneously without extending the gauge sector and the fermionic sector of the SM . In our framework, these anomalies can be resolved by a new CP-even light scalar state that resides in the $\mathcal{O}(10)$ $\mathrm{MeV}$ to $\mathcal{O}(1)-\mathrm{GeV}$ mass range, which provides correct sizes and signs for these deviations due to one-loop and two-loop dominance for the muon and the electron, respectively. A light scalar of similar type has been studied in the context of muon anomalous magnetic moment $[11,12]$. As we will show, in our setup such a light scalar can be emerge from a second Higgs doublet, and subject to a number of various experimental constraints and it can still simultaneously incorporate the deviations observed in the muon and the electron anomalous magnetic moments. 


\section{MODEL}

Our UV-complete theory is the well-motivated two Higgs-doublet-model (2HDM). The most general scalar potential of 2HDM written in the Higgs-basis is given by [13]:

$$
\begin{aligned}
V & =m_{11}^{2} H_{1}^{\dagger} H_{1}+m_{22}^{2} H_{2}^{\dagger} H_{2}-\left\{m_{12}^{2} H_{1}^{\dagger} H_{2}+\text { h.c. }\right\}+\frac{\lambda_{1}}{2}\left(H_{1}^{\dagger} H_{1}\right)^{2}+\frac{\lambda_{2}}{2}\left(H_{2}^{\dagger} H_{2}\right)^{2}+\lambda_{3}\left(H_{1}^{\dagger} H_{1}\right)\left(H_{2}^{\dagger} H_{2}\right) \\
& +\lambda_{4}\left(H_{1}^{\dagger} H_{2}\right)\left(H_{2}^{\dagger} H_{1}\right)+\left\{\frac{\lambda_{5}}{2}\left(H_{1}^{\dagger} H_{2}\right)^{2}+\text { h.c. }\right\}+\left\{\left[\lambda_{6}\left(H_{1}^{\dagger} H_{1}\right)+\lambda_{7}\left(H_{2}^{\dagger} H_{2}\right)\right] H_{1}^{\dagger} H_{2}+\text { h.c. }\right\} .
\end{aligned}
$$

We work in the $\mathrm{CP}$-conserving limit and take all the parameters to be real. Note that in this basis, only $H_{1}$ has non-zero VEV, and these fields can be parametrized as:

$$
H_{1}=\left(\begin{array}{c}
G^{+} \\
\frac{v+H_{1}^{0}+i G^{0}}{\sqrt{2}}
\end{array}\right), H_{2}=\left(\begin{array}{c}
H^{+} \\
\frac{H_{2}^{0}+i A^{0}}{\sqrt{2}}
\end{array}\right)
$$

Here $G^{+}$and $G^{0}$ are the Goldstone bosons eaten up by the gauge bosons after the EW symmetry is broken. In our study, we work in the alignment limit, in this limit $H_{1}^{0} \approx h$ is the SM Higgs and almost decouple from the other CP-even state $H_{2}^{0} \approx H$. The relevant Yukawa Lagrangian of the model can be written as:

$$
-\mathcal{L}_{Y} \supset\left[Y_{\ell, i j}^{H^{0}} H^{0}+i Y_{\ell, i j}^{A^{0}} A^{0}\right] \bar{\ell}_{L i} \ell_{R j}+Y_{\ell, i j}^{H^{+}} \bar{v}_{L i} \ell_{R j} H^{+} \sqrt{2}+\text { h.c. }
$$

here, $Y_{\ell}^{H^{0}}=Y_{\ell}^{A^{0}}=Y_{\ell}^{H^{+}}=Y_{\ell}$. We assume a diagonal texture for this Yukawa matrix and take them to be real.

In our setup, the dominant contributions to the lepton anomalous magnetic moments are shown in Fig. 1. The full one-loop contributions to $\Delta a_{\ell}$ arising from the charged, CP-even, and CP-odd scalars are given by [14]:

$$
\begin{gathered}
\Delta a_{1, \ell}^{H^{+}}=\frac{-Q_{H^{+}}\left(Y_{\ell}^{H^{+}}\right)^{2}}{4 \pi^{2}} \int_{0}^{1} d x \frac{x^{2}(x-1)}{x^{2}+x\left(z_{H^{+}}^{2}-1\right)}, \\
\Delta a_{1, \ell}^{\phi^{0}}=\frac{-1}{8 \pi^{2}} \sum_{f} \sum_{\phi^{0}=}^{H, A} Q_{f}\left|Y_{\ell}^{\phi^{0}}\right|^{2} \int_{0}^{1} d x \frac{x^{2}\left(1-x \pm z_{f}\right)}{x^{2}+x\left(z_{f}^{2}-1\right)+z_{\phi^{0}}^{2}(1-x)}, \\
z_{H^{+}}=\frac{m_{H^{+}}}{m_{\ell}}, z_{f}=\frac{m_{f}}{m_{\ell}}, z_{\phi^{0}}=\frac{m_{\phi^{0}}}{m_{\ell}},
\end{gathered}
$$

In the above equation, + and - corresponds to the cases $\phi^{0}=H$ and $\phi^{0}=A$, respectively. Within our set-up, the neutral scalars with the help of fermion loops can contribute to $\Delta a_{\ell}$ via a two-loop Barr-Zee diagram $[15,16]$ as shown in Fig. 1 (diagram on the right). The corresponding two-loop contributions to $\Delta a_{e, \mu}$ can be written as:

$$
\begin{aligned}
& \Delta a_{2, \ell}^{\phi^{0}}=\frac{\alpha}{8 \pi^{3}} m_{\ell} Y_{\ell}^{\phi^{0}} \sum_{f} \sum_{\phi^{0}=}^{H, A} \frac{N_{f}^{c} Q_{f}^{2} Y_{f}^{\phi^{0}}}{m_{f}} F_{\phi^{0}}\left[\frac{m_{f}^{2}}{m_{\phi^{0}}^{2}}\right] \\
& F_{\phi^{0}}\left[z_{\phi^{0}}\right]=z_{\phi^{0}} \int_{0}^{1} d x \frac{w_{\phi^{0}}}{x(1-x)-z_{\phi^{0}}} \ln \frac{x(1-x)}{z_{\phi^{0}}} \\
& w_{H}=2 x(1-x)-1, w_{A}=1 .
\end{aligned}
$$

In the above equation, the sum over the internal fermions is taken over $f=e, \mu, \tau$.

We are interested in an interesting regime of the $2 \mathrm{HDM}$ where the $\mathrm{CP}$-even state $H$, emerging from the second Higgs doublet remains sufficiently light compared to its partners. In our scenario, a mass splitting of this type is essential for a concurrent explanation of $\Delta a_{\mu}$ and $\Delta a_{e}$. Therefore, in this scheme, only the contribution of the light state to the $\Delta a_{e, \mu}$ is significant, since our case corresponds to $m_{H^{+}}=m_{A} \gg m_{H}$.

In Fig. 2, we show the parameter space in Yukawa coupling vs mass of the light scalar $\left(m_{H}\right)$ plane consistent with both $\Delta a_{e}$ and $\Delta a_{\mu}$. In making these plots, we fix the tau Yukawa coupling to be $y_{\tau}=0.1$, which is allowed by the experiment data to be discussed later in the text. From Fig. 2, it is clear that there exist a significant region where one can simultaneously explain the anomalies in the lepton anomalous magnetic moments. However, a significant portion of this parameter space is constrained by various experiments, which we will be discussing in the next section. 

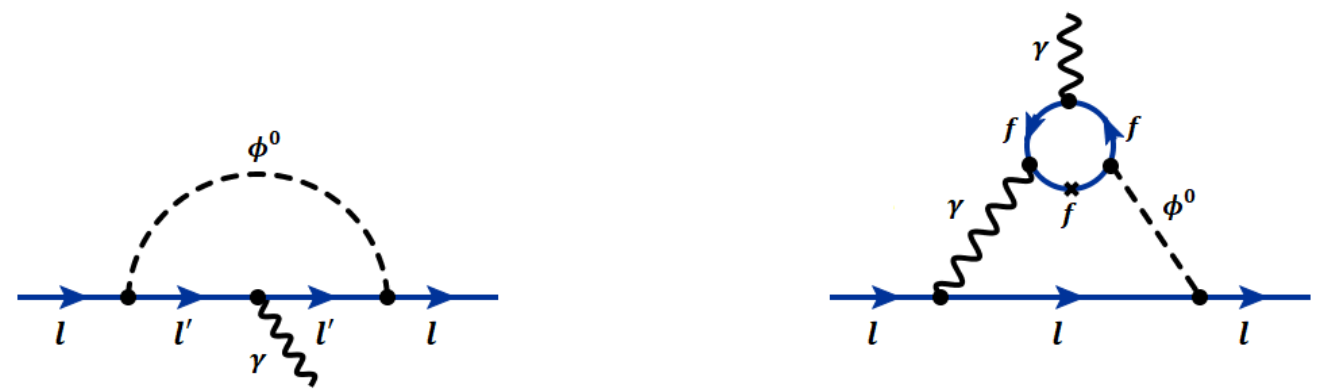

FIGURE 1: One-loop (left) and two-loop (right) contributions to lepton anomalous magnetic moments arising from beyond-SM neutral scalars. The one-loop contribution due to the charged scalar is not presented here.
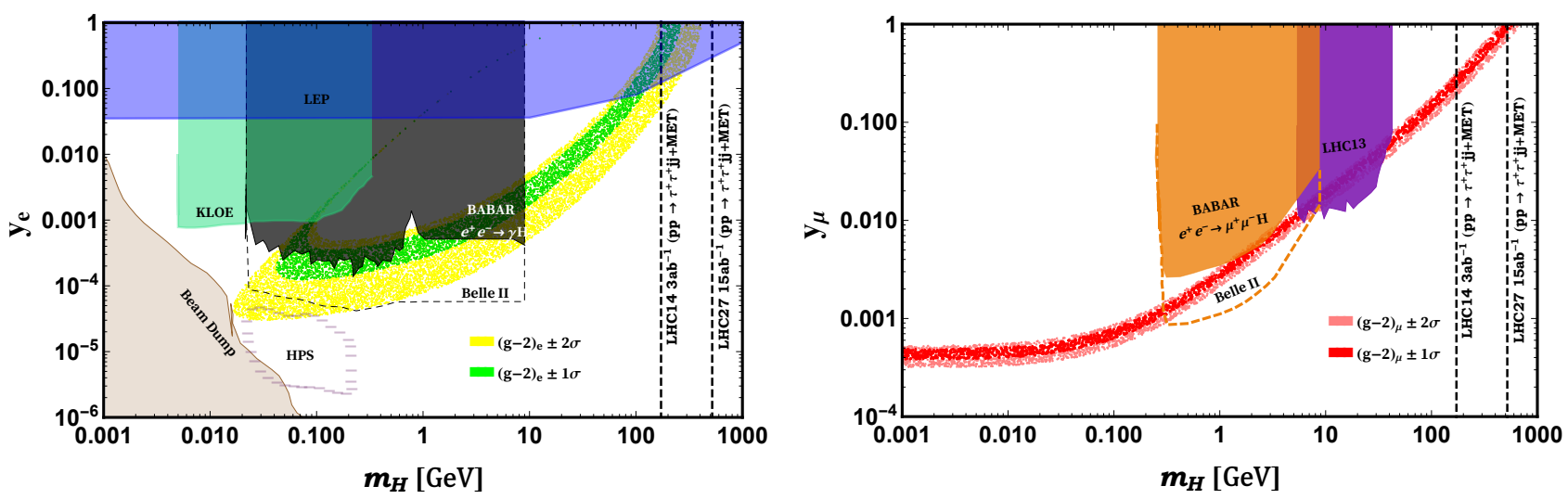

FIGURE 2: The parameter space in Yukawa coupling $\left(y_{l}\right.$, whrere $l=e$ or $\left.\mu\right)$ vs mass $\left(m_{H}\right)$ plane consistent with both the electron and muon anomalous magnetic moments. The green (red) and yellow (pink) regions represent the experimental $1 \sigma$ and $2 \sigma$ bands for the electron (muon) anomalous magnetic moment $\Delta a_{e}\left(\Delta a_{\mu}\right)$. The color shaded regions with solid boundary denote the excluded parameter space by current experiments. The projected sensitivities for the signal $p p \rightarrow H^{ \pm} H^{ \pm} j j \rightarrow \tau^{ \pm} \tau^{ \pm} j j+E_{T}$ at the LHC for centre of mass energy $14 \mathrm{TeV}$ with integrated luminosity $\mathcal{L}=3 \mathrm{ab}^{-1}$ and also for the centre of mass energy $27 \mathrm{TeV}$ with integrated luminosity $\mathcal{L}=15 \mathrm{ab}^{-1}$ are shown by black dashed vertical lines.

\section{EXPERIMENTAL CONSTRAINTS AND FUTURE PROSPECTS}

As aforementioned, a light scalar of mass $m_{H}<\mathrm{TeV}$, having sizable couplings to the SM charged leptons is subject to diverse experimental constraints. One of the stringent constrain is from the Electron beam-dump experiments [17]. In these experiments light scalars can be produced via Bremsstrahlung-like processes: $e+N \rightarrow e+N+H$. The lack of such events at the electron beamdump experiments provide stringent constraints on the mass of the light scalar and its corresponding couplings to the electrons.

There are several experiments that search for the presence of dark-photons and their null observations can be translated to provide stringent constraints on the allowed parameter space of light scalars. KLOE collaboration [18] searches for the dark-photons $A_{d}$ through the process: $e^{+} e^{-} \rightarrow \gamma A_{d}$, with $A_{d} \rightarrow e^{+} e^{-}$. The lack of such signals at this experiment can be used to set constraints on the light scalars that have coupling with the electrons, which is indicated by cyan-shaded region in Fig. 2 . Through a similar process, the BaBar collaboration [19] also searches for the dark-photons with $A_{d} \rightarrow \ell^{+} \ell^{-}$. By recasting the results from BaBar, this provides exclusion regions in the light scalar mass and Yukawa coupling plane, which is depicted by a light-black shaded region in Fig. 2. For a scalar mass $m_{H}>200 \mathrm{MeV}$ the dark-boson searches at the BaBar [20] can be used to impose limits on $H \mu^{+} \mu^{-}$coupling via $e^{+} e^{-} \rightarrow \mu^{+} \mu^{-} H$ process. We recast this result for our scenario, which is shown as light brown shaded region in Fig. 2.

LEP experiment [21] impose stringent constraints on $e^{+} e^{-} \rightarrow f \bar{f}$ processes. The most constraining process is the one with electrons in the final states. The obtained LEP bounds for our model is projected in Fig. 2 in blue-shaded region. As far as the LHC bounds, most of the searches for heavy neutral scalars are done in the context of either MSSM or generic 2HDM, which are not directly applicable in our scenario since, $\phi^{0}$ has negligible couplings to quarks, and therefore, cannot be produced via gluon fusion. However, LHC bounds on neutral scalars come out to be weaker than the LEP bounds as discussed above due to its leptophilic nature. Apart from these constraints from the collider, exotic $Z$ decay searches of the type $Z \rightarrow 4 \mu$ at LHC put strong constraint on the mass of the light scalar and its corresponding coupling to the muons, which is plotted as a purple region in Fig. 2. 


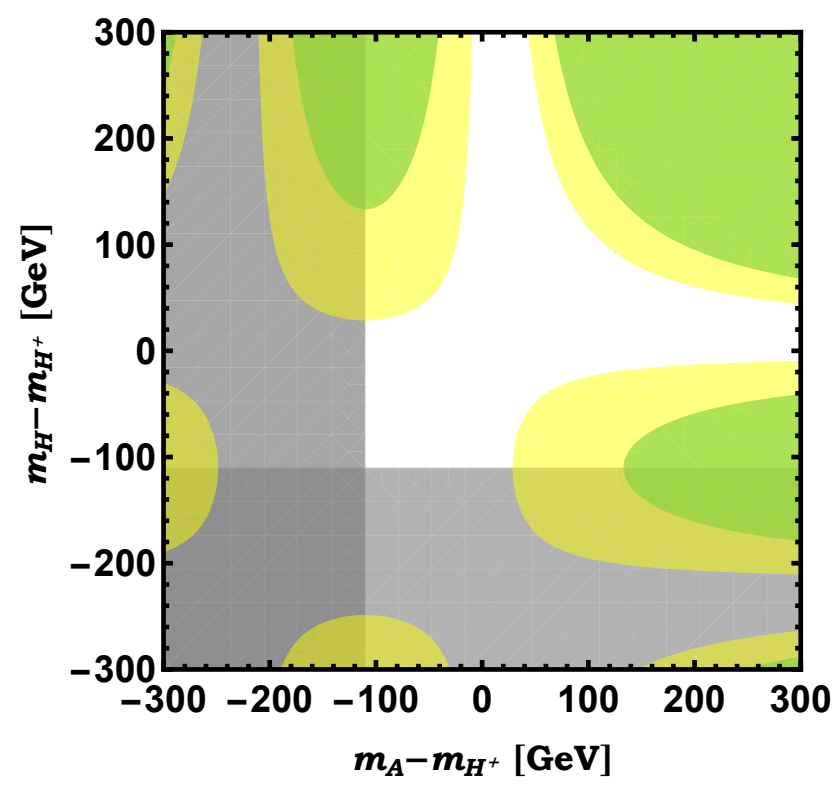

FIGURE 3: Scalar mass splittings allowed by the $T$ parameter constraint in the 2HDM. The yellow and green shaded regions represent the $1 \sigma$ and $2 \sigma$ exclusion regions from the $T$ parameter constraint [23]. The horizontal and vertical grey shaded regions indicate the positivity criteria for $m_{H}>0$ and $m_{A}>0$, respectively. Here, we set $m_{H^{ \pm}}=110 \mathrm{GeV}$.

EW precision data can impose strong constraints on our setup. In the alignment limit, the $T$ parameter in the $2 \mathrm{HDM}$ can be expressed as [22]:

$$
T=\frac{1}{16 \pi s_{W}^{2} M_{W}^{2}}\left\{\mathcal{F}\left(m_{H^{+}}^{2}, m_{H}^{2}\right)+\mathcal{F}\left(m_{H^{+}}^{2}, m_{A}^{2}\right)-\mathcal{F}\left(m_{H}^{2}, m_{A}^{2}\right)\right\},
$$

where the symmetric function $\mathcal{F}$ is given by

$$
\mathcal{F}\left(m_{1}^{2}, m_{2}^{2}\right) \equiv \frac{1}{2}\left(m_{1}^{2}+m_{2}^{2}\right)-\frac{m_{1}^{2} m_{2}^{2}}{m_{1}^{2}-m_{2}^{2}} \ln \left(\frac{m_{1}^{2}}{m_{2}^{2}}\right) .
$$

The corresponding region plot is shown in Fig. 3. The yellow and green shaded regions indicate the $1 \sigma$ and $2 \sigma$ exclusion regions from the $T$ parameter constraint, respectively. The horizontal and vertical gray shaded regions corresponds to the positivity criteria for $m_{H}>0$ and $m_{A}>0$, respectively. From this figure it is apparent that our scenario: $m_{H}^{2} \ll m_{H^{+}}^{2}=m_{A}^{2} \sim \mathcal{O}(110) \mathrm{GeV}$ is well consistent with the EW precision constraints.

Now we discuss the testability of the proposed scenario in the upcoming experiments. As we discussed earlier, explanations of the experimental data of $\Delta a_{e, \mu}$ solely depend on the existence of a light CP-even scalar. This scenario can be tested at the LHC by looking at the novel process $p p \rightarrow H^{ \pm} H^{ \pm} j \rightarrow \tau^{ \pm} \tau^{ \pm} j j+\mathbb{E}_{T}$, and the corresponding representative Feynman diagram is presented in Fig. 4. It is interesting to note that if the mass splitting between the CP-even and CP-odd neutral scalars is turned off, then the amplitude for this process will be exactly zero. Correspondingly, our scenario will fail to explain the lepton anomalous magnetic moments, since a large mass splitting is essential to properly incorporate $\Delta a_{e, \mu}$ data as discussed above. Hence, observed deviations in the lepton anomalous magnetic moments are directly correlated with the signal $p p \rightarrow \tau^{ \pm} \tau^{ \pm} j j+E_{T}$ in our set-up. Due to this complementarity, this particular explanation of the electron and the muon $g-2$ within the 2HDM can be tested by this novel same sign charge lepton process. This same-sign charged lepton signature via vector-boson fusion process at the LHC has been studied extensively in Ref. [24], although in a different context. We recast this analysis for our case and obtain the projected sensitivity for the signal $p p \rightarrow H^{ \pm} H^{ \pm} j j \rightarrow \tau^{ \pm} \tau^{ \pm} j j+E_{T}$ at the LHC. These projected sensitivities are shown in Fig. 2 by black dashed lines.

\section{SUMMARY AND DISCUSSION}

Motivated by the recent precise measurement of the electron anomalous magnetic moment $a_{e}$, which shows a significant deviation from the SM prediction, together with the intriguing deviation observed in the muon anomalous magnetic moment $a_{\mu}$, here we present a novel scenario consisting of a light neutral scalar $H$ that is behind the origin of both these anomalies. By properly taking into account theoretical and all existing experimental constraints, we have shown that a wide range of parameter space $\mathcal{O}(10) \mathrm{MeV}$ $\leq m_{H} \leq \mathcal{O}(1) \mathrm{GeV}$ is still allowed, which provides correct sizes and signs for both the $a_{e}$ and $a_{\mu}$. We have demonstrated how such a light $\mathrm{CP}$-even scalar naturally arises from general 2HDM and serves the required purpose. Our model predicts that the light scalar $H$ must be accompanied by nearly degenerate charged scalar $H^{+}$and a pseudoscalar $A$ that have masses of the order of the EW symmetry breaking scale. A complementarity test of this scenario at the LHC by seeking the novel process $p p \rightarrow H^{ \pm} H^{ \pm} j i \rightarrow$ $l^{ \pm} l^{ \pm} j j+E_{T}$ via same-sign pair production of charged Higgs bosons is also discussed. 


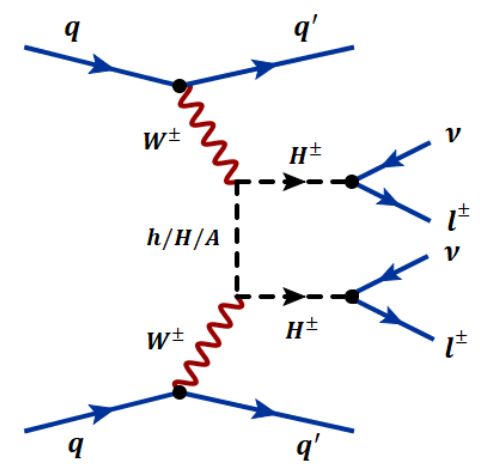

FIGURE 4: Representative Feynman diagram for the signal $p p \rightarrow \tau^{+} \tau^{+} j j+E_{T}$ at the LHC.

\section{References}

[1] J. S. Schwinger, Phys. Rev. 73, 416-417 (1948) doi:10.1103/PhysRev.73.416

[2] G. W. Bennett et al. [Muon g-2], Phys. Rev. D 73, 072003 (2006) doi:10.1103/PhysRevD.73.072003 [arXiv:hep-ex/0602035 [hep-ex]].

[3] R. H. Parker, C. Yu, W. Zhong, B. Estey and H. Müller, Science 360, 191 (2018) doi:10.1126/science.aap7706 [arXiv:1812.04130 [physics.atom$\mathrm{ph}]]$.

[4] H. Davoudiasl and W. J. Marciano, Phys. Rev. D 98, no.7, 075011 (2018) doi:10.1103/PhysRevD.98.075011 [arXiv:1806.10252 [hep-ph]].

[5] J. Liu, C. E. M. Wagner and X. P. Wang, JHEP 03, 008 (2019) doi:10.1007/JHEP03(2019)008 [arXiv:1810.11028 [hep-ph]].

[6] I. Doršner, S. Fajfer and S. Saad, Phys. Rev. D 102, no.7, 075007 (2020) doi:10.1103/PhysRevD.102.075007 [arXiv:2006.11624 [hep-ph]].

[7] K. F. Chen, C. W. Chiang and K. Yagyu, JHEP 09, 119 (2020) doi:10.1007/JHEP09(2020)119 [arXiv:2006.07929 [hep-ph]].

[8] S. Jana, P. K. Vishnu, W. Rodejohann and S. Saad, Phys. Rev. D 102, no.7, 075003 (2020) doi:10.1103/PhysRevD.102.075003 [arXiv:2008.02377 [hep-ph]].

[9] A. Crivellin and M. Hoferichter, PoS ALPS2019, 009 (2020) doi:10.22323/1.360.0009 [arXiv:1905.03789 [hep-ph]].

[10] S. Jana, V. P. K. and S. Saad, Phys. Rev. D 101, no.11, 115037 (2020) doi:10.1103/PhysRevD.101.115037 [arXiv:2003.03386 [hep-ph]].

[11] K. S. Babu, S. Jana, M. Lindner and V. P. K, [arXiv:2104.03291 [hep-ph]].

[12] V. Brdar, S. Jana, J. Kubo and M. Lindner, [arXiv:2104.03282 [hep-ph]].

[13] K. S. Babu and S. Jana, JHEP 02, 193 (2019) doi:10.1007/JHEP02(2019)193 [arXiv:1812.11943 [hep-ph]].

[14] J. P. Leveille, Nucl. Phys. B 137, 63-76 (1978) doi:10.1016/0550-3213(78)90051-2

[15] J. D. Bjorken and S. Weinberg, Phys. Rev. Lett. 38, 622 (1977) doi:10.1103/PhysRevLett.38.622

[16] S. M. Barr and A. Zee, Phys. Rev. Lett. 65, 21-24 (1990) [erratum: Phys. Rev. Lett. 65, 2920 (1990)] doi:10.1103/PhysRevLett.65.21

[17] M. Davier and H. Nguyen Ngoc, Phys. Lett. B 229, 150-155 (1989) doi:10.1016/0370-2693(89)90174-3

[18] A. Anastasi, D. Babusci, G. Bencivenni, M. Berlowski, C. Bloise, F. Bossi, P. Branchini, A. Budano, L. Caldeira Balkeståhl and B. Cao, et al. Phys. Lett. B 750, 633-637 (2015) doi:10.1016/j.physletb.2015.10.003 [arXiv:1509.00740 [hep-ex]].

[19] J. P. Lees et al. [BaBar], Phys. Rev. Lett. 113, no.20, 201801 (2014) doi:10.1103/PhysRevLett.113.201801 [arXiv:1406.2980 [hep-ex]].

[20] J. P. Lees et al. [BaBar], Phys. Rev. D 94, no.1, 011102 (2016) doi:10.1103/PhysRevD.94.011102 [arXiv:1606.03501 [hep-ex]].

[21] t. Electroweak [LEP, ALEPH, DELPHI, L3, OPAL, LEP Electroweak Working Group, SLD Electroweak Group and SLD Heavy Flavor Group], [arXiv:hep-ex/0312023 [hep-ex]].

[22] W. Grimus, L. Lavoura, O. M. Ogreid and P. Osland, J. Phys. G 35, 075001 (2008) doi:10.1088/0954-3899/35/7/075001 [arXiv:0711.4022 [hepph]].

[23] M. Tanabashi et al. [Particle Data Group], Phys. Rev. D 98, no.3, 030001 (2018) doi:10.1103/PhysRevD.98.030001

[24] M. Aiko, S. Kanemura and K. Mawatari, Phys. Lett. B 797, 134854 (2019) doi:10.1016/j.physletb.2019.134854 [arXiv:1906.09101 [hep-ph]]. 